\title{
MASS ESTIMATION OF IMPACTING OBJECTS AGAINST A STRUCTURE USING AN ARTIFICIAL NEURAL NETWORK WITHOUT CONSIDERATION OF BACKGROUND NOISE
}

\author{
SUNG-HWAN SHIN*, JIN-HO PARK, DOO-BYUNG YOON, and YOUNG-CHUL CHOI \\ Advanced Condition Monitoring and Diagnostics Laboratory \\ Korea Atomic Energy Research Institute (KAERI), Daejeon (305-353), Korea \\ "Corresponding author. E-mail : soulshin@kaeri.re.kr
}

Received November 17, 2010

Accepted for Publication March 09, 2011

It is critically important to identify unexpected loose parts in a nuclear reactor pressure vessel, since they may collide with and cause damage to internal structures. Mass estimation can provide key information regarding the kind as well as the location of loose parts. This study proposes a mass estimation method based on an artificial neural network (ANN), which can overcome several unresolved issues involved in other conventional methods. In the ANN model, input parameters are the discrete cosine transform (DCT) coefficients of the auto-power spectrum density (APSD) of the measured impact acceleration signal. The performance of the proposed method is then evaluated through application to a large-sized plate and a 1/8-scaled mockup of a reactor pressure vessel. The results are compared with those obtained using a conventional method, the frequency ratio (FR) method. It is shown that the proposed method is capable of estimating the impact mass with $30 \%$ lower relative error than the FR method, thus improving the estimation performance.

KEYWORDS : Mass Estimation, Artificial Neural Network, Discrete Cosine Transform, Loose Part Monitoring, Reactor Pressure Vessel

\section{INTRODUCTION}

In a large-sized vessel type structure such as the reactor pressure vessel in a nuclear power plant, an unexpected loose part can cause serious problems during plant operation. The loose part may collide against the inner wall or the internal structures of the vessel, which may give rise to negative effects on the safety and health of the structure. Accordingly, for the maintenance of a nuclear power plant, monitoring whether there are any loose parts in the reactor pressure vessel is an important task and one of the functions of the Nuclear Steam Supply System Integrity Monitoring System (NIMS) [1].

Generally, parts that become loose are metal pieces such as bolts, nuts, fragments, sleeves, and so on. There are two major goals in the task of monitoring the presence of loose parts in a large-sized vessel: localization, that is, finding where the loose part is, and mass estimation, estimating the weight of the loose part. For various types of structures, several different methods such as the hyperbola intersection method, the circle intersection method, the triangular intersection method, and modified and improved versions of these approaches have yielded successful localization [1-4]. In contrast, mass estimation methods using accelerometers or acoustic emission sensors [5,6] still require further improvement.

Conventional methods for mass estimation exploit the impact signal generated when a loose part collides against a structure. These methods have some shortcomings that reduce the accuracy and reliability of the estimation. For example, an analytic method based on Hertz's impact theory [7] suffers from an uncertainty problem that should be resolved. Furthermore, it is assumed that the two colliding solid objects (two solid spheres or one solid sphere and one plate) are rigid, which is usually not true in the real world. Another approach is the frequency ratio (FR) method [8]. FR is defined as follows:

$$
F R=\frac{\int_{f_{t, \text { min }}}^{f_{L, \text { max }}} S(f) d f}{\int_{f_{H, \text { min }}}^{f_{H, \max }} S(f) d f}
$$

where $f$ is the frequency, $S(f)$ is the auto-power spectrum density (APSD) of the measured impact signal, $f_{L, \max }$ and $f_{L, \text { min }}$ denote the maximum and minimum frequencies of the low frequency band, respectively, and $f_{H \cdot \max }$ and $f_{H \cdot \min }$ are the maximum and minimum frequencies of the high frequency band, respectively. Here, the low frequency band is the frequency range that is subjected to impact 
(a)

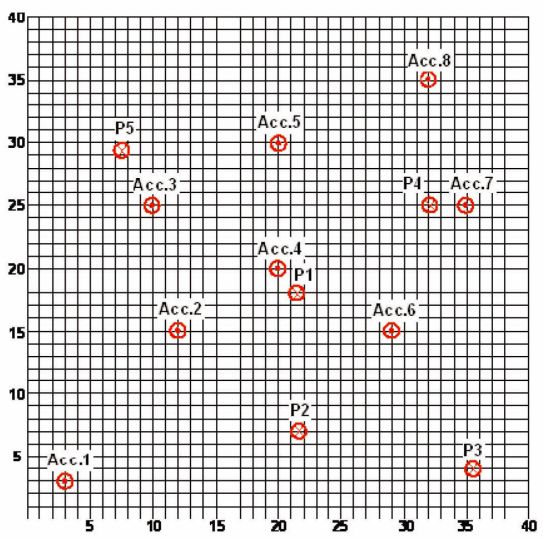

(b)

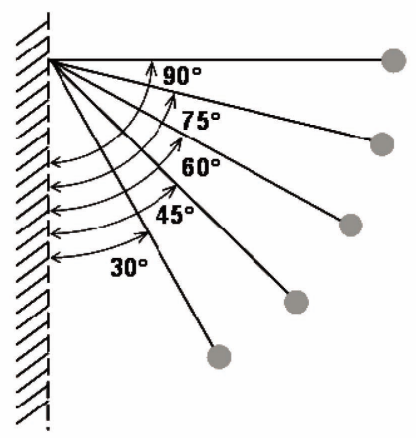

Fig. 1. (a) Positions of Accelerometers (Acc. \#) and Excitation Points (P \#) on a Large-sized Plate $(2 \mathrm{~m} \times 2 \mathrm{~m} \times 0.01 \mathrm{~m})$ and $(\mathrm{b})$ the Method to Impact a Solid Sphere Against the Plate with Different Impact Energy

mass. The high frequency band is the range wherein features of the used sensor are dominant and the APSD pattern is rarely changed by external excitation. The FR method has a wide uncertainty range of approximately 0.7 decade for the estimated mass [8]. In addition, the user should select how to determine the maximum and minimum frequency limits for dividing the whole frequency range into low and high ranges.

The present study develops a mass estimation method using an artificial neural network (ANN) and a discrete cosine transform (DCT) in order to estimate the mass of loose parts with improved accuracy and reliability. Figedy and Oksa [9] stated that using an ANN with 4 input parameters, the mean values of the APSD in four defined frequency bands, could improve the estimation performance. Unfortunately, they did not establish a criterion to determine the number of frequency bands.

In this study, first, a method of extracting the pattern of the APSD curve of the impact signal is studied. There are several kinds of nuisance variables that affect the pattern of the APSD curve such as the transmission properties of the structure, loose part mass, sensor position, impact energy (or velocity), loose part shape, coupling between the structure and the other medium (e.g. fluid), and so on. Here, the effects of loose part shape and coupling between media are not considered due to their variation and difficulty of measurement.

Second, an ANN model is designed by using quantitative variables expressing the distribution pattern of the APSD obtained from each sensor as input and the actual mass of an impacting object as output. Next, the FR method is applied to the impact signals used for the second stage. Finally, through a comparison between the results obtained from the proposed method and the FR method, the performance of the mass estimation method using an ANN is examined. A large-sized plate and a 1/8-scaled mockup of a reactor pressure vessel are employed as the application structures.

\section{FREQUENCY CHARACTERISTICS OF IMPACT SIGNAL}

It is well known that the mass of a loose part considerably changes the APSD curve of an impact signal [10]. For this reason, the APSD of an impact signal is considered an important factor for estimating a loose part mass. To confirm this and to see the relation between the impact condition and APSD in greater detail, an experiment to measure impact signals was conducted for different impact conditions. The structure used for the experiment was a large-sized steel plate $(2 \mathrm{~m}$ (width) $\times 2 \mathrm{~m}$ (height) $\times 0.01 \mathrm{~m}$ (thickness) ).

In the experiment, 8 accelerometers were positioned at arbitrary points on the plate and impact excitation was applied at 5 fixed points in order to examine the effects of transmission properties and sensor position. All accelerometers have a $55 \mathrm{kHz}$ mounted resonance frequency. Fig. 1(a) shows the positions of the accelerometers and excitation points. For the excitation, 3 solid steel spheres with different masses, $112.7 \mathrm{~g}, 45.8 \mathrm{~g}$, and $9.4 \mathrm{~g}$, were used to assess the effects of impact mass. Impact energy was adjusted by changing the height at which the sphere was released among 5 points, as shown in Fig.1(b).

First, the relation between the impact mass and the APSD is examined. Fig. 2 compares APSD curves of impact signals for different masses at the collision point. The APSD curves show a pattern consisting of a broad main peak (or lobe) in the low frequency range and other minor peaks. As stated earlier, the APSD changes considerably with the impact mass. The main peak 

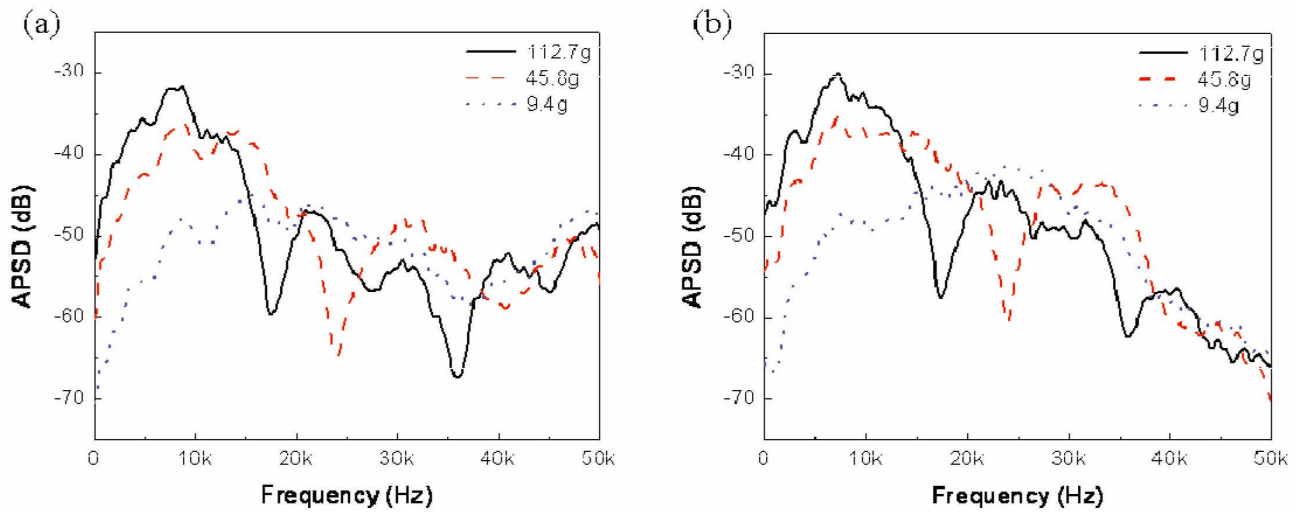

Fig. 2. Comparisons of the Auto-power Spectrum Density (APSD) Curves Measured at (a) Acc. 1 and (b) Acc. 4 According to Impact Mass when an Impact was Made at P1 on the Plate

(a)

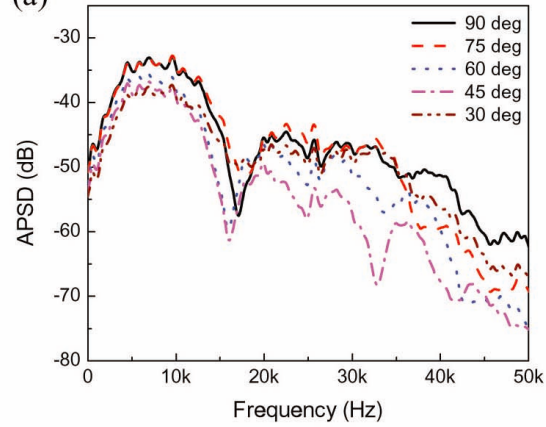

(b)

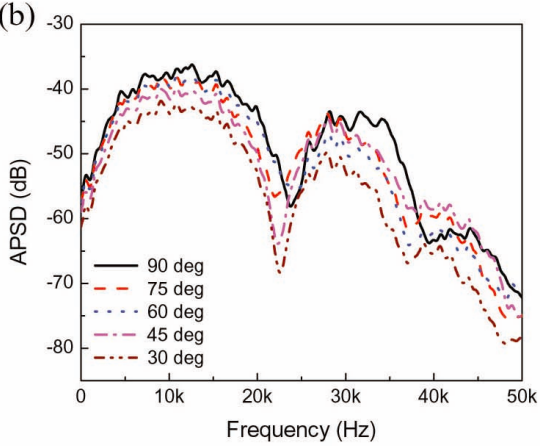

(c)

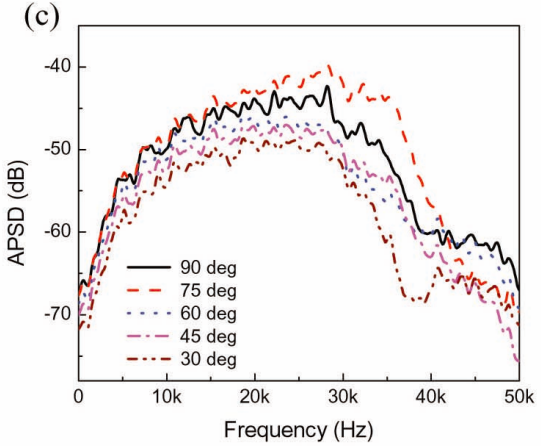

Fig. 3. Comparisons of the Auto-power Spectrum Density (APSD) Curves Measured at Acc.4 According to the Impact Energy when Impact Masses of (a) 112.7g, (b) 45.8g, and (c) 9.4g were Impacted at P4 on the Plate

moves to lower frequency as the impact mass increases. On a theoretical basis, the main peak is related to the dominant frequency $\left(f_{\text {main }}\right)$ that occurs when the sphere collides against the plate and is determined by the contact duration $\left(T_{\text {contact }}\right)$ given by Hertz's theory as follows [10]:

$$
f_{\text {main }}=\frac{1}{T_{\text {contact }}} \propto m^{-2 / 5}
$$

where $m$ is the mass of the sphere. In addition, the bandwidth of the main peak becomes narrower when the mass is heavier.

Second, APSD curves for different levels of impact energy are compared in Fig. 3. For the same impact mass, the overall patterns of the APSD are nearly the same regardless of the energy levels. However, in some cases, the main peak slightly shifts to a low frequency when the impact energy is low, that is, the release height is low. For example, the bandwidth of the main peak is narrowed by about $6 \%$ in Figs. 3(a) and (b) when the release height is decreased from $90^{\circ}$ to $45^{\circ}$ in Fig. 1(b). This means that the object mass can be estimated to be heavier than the actual mass when it collides against a wall with low energy or velocity.

Next, Fig. 4 shows APSD curves for different impact points. It is observed in these figures that there is no appreciable change in the main peak with different impact points. However, the positions and bandwidths of minor peaks in the high frequency range vary slightly without any specific tendency.

Finally, Fig. 5 compares the relation between the measuring point and the APSD. The main peaks of the APSD curves for different points have nearly the same pattern. Strictly speaking, the main peak of the impact signal measured from Acc.1, which was located nearest to the impact point, P1, has a complex form. However, its overall pattern is not substantially different from that of the others in spite of the existence of small peaks. Contrary to the main peak, the other minor peaks show some difference in shape depending on their positions.

In summary, the APSD of the impact signals is mainly influenced by the impact mass if the shape of the impact object is fixed and possible coupling effects 
(a)

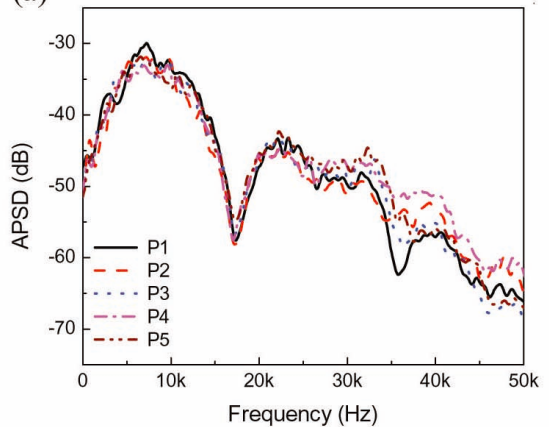

(b)

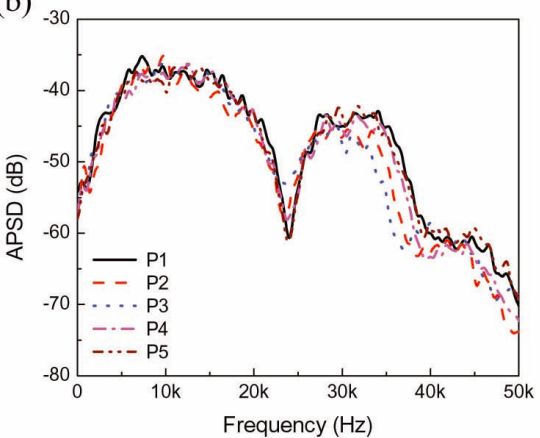

(c)

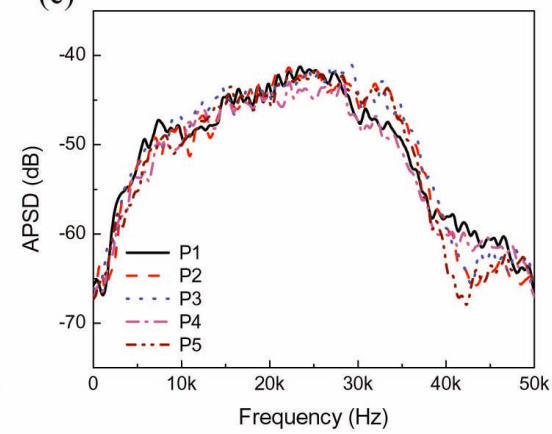

Fig. 4. Comparisons of Auto-power Spectrum Density (APSD) Curves Measured at Acc.4 According to the Impact Point on the Plate when the Released Angle of Impact Masses of (a) $112.7 \mathrm{~g}$, (b) $45.8 \mathrm{~g}$, and (c) $9.4 \mathrm{~g}$ was $90^{\circ}$

(a)

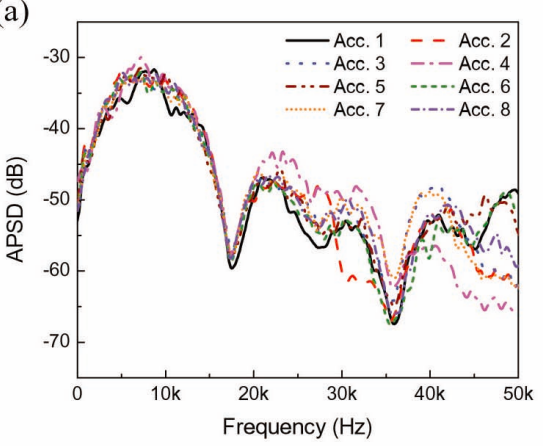

(b)

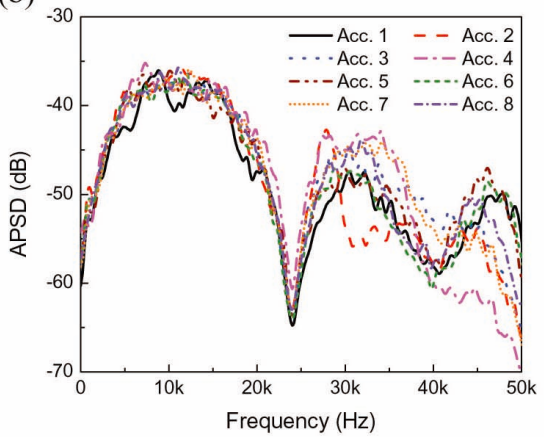

(c)

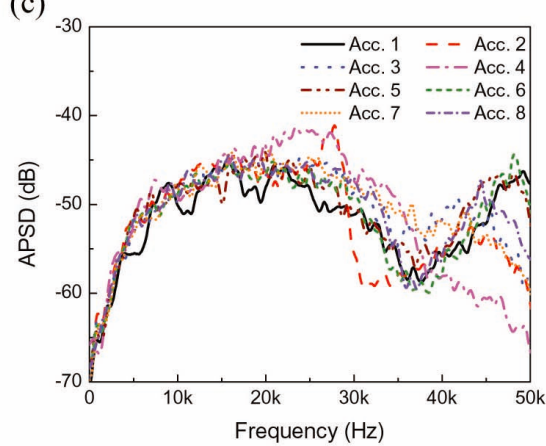

Fig. 5. Comparisons of Auto-power Spectrum Density (APSD) Curves According to the Measuring Point when the Released Angle of Impact Masses of (a) $112.7 \mathrm{~g}$, (b) $45.8 \mathrm{~g}$, and (c) $9.4 \mathrm{~g}$ was $90^{\circ}$ and the Impact Point was P1 on the Plate

between the structure and other medium are neglected. It appears that the impact energy is another factor that causes changes to the APSD. In addition, variation of the impact point and the measuring points does not cause appreciable changes in the main peak shape of the APSD for the plates used in this comparison. For much larger structures where the distance between two measuring points or impact and measuring points is far, the effect of the impact and measuring points may need to be considered as a nuisance variable.

\section{DESIGN OF ARTIFICIAL NEURAL NETWORK MODEL}

This study employs an ANN model to estimate the mass of an impacting object. Among the deterministic models for detecting and recognizing a specific signal pattern, the ANN has been widely used for cases in which the input-output relationship is strongly nonlinear $[9,10]$. In general, the ANN has a strong theoretical background and performs feature mapping, with high accuracy, from input parameters in an ambiguous form to the expected domains [13].

\subsection{Feature Extraction}

As in other estimation or recognition methods, the most important task in the design of the ANN model is to extract an optimal feature vector, that is, input parameters that represent the characteristics of the objects under consideration as accurately as possible. In this study, the APSD of an impact signal generated when a loose part collides against a wall or a structure is taken as a strong candidate for the feature vector, since APSDs of impact signals are remarkably different depending on the impact mass, as shown in Chapter 2. Unfortunately, it is not reasonable to use a whole set of raw APSDs as the feature vector due to the large amount of data. Using a whole set of raw APSDs will increase the complexity of the ANN model and lead to deterioration of its estimation performance. For this reason, a portion of the information in the APSD such as the FR value [8] or four-band 
(a)

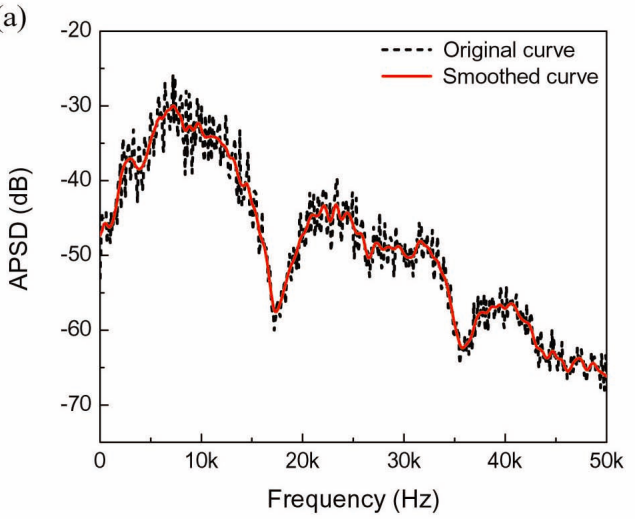

(c)

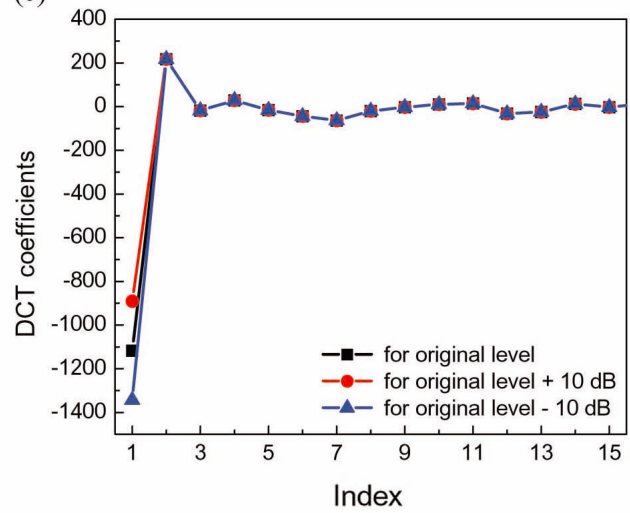

(b)

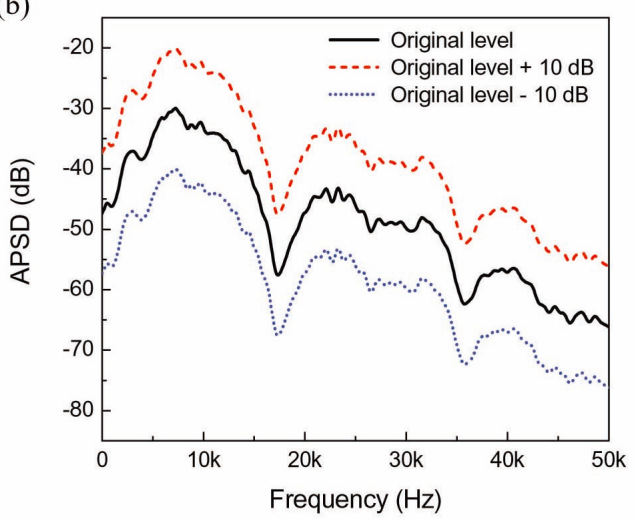

(d)

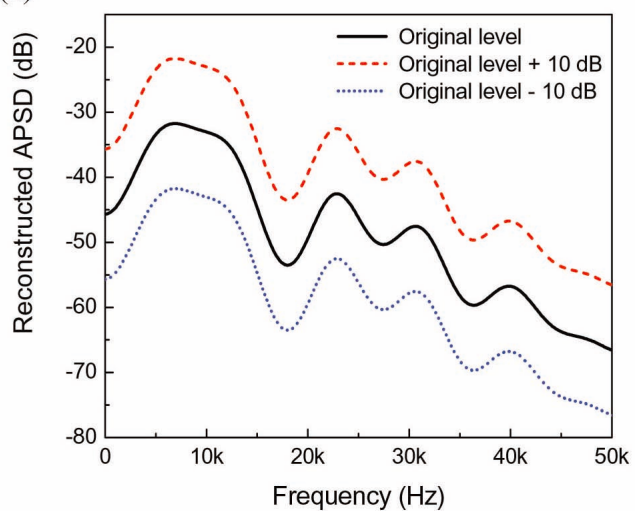

Fig. 6. Comparisons Between/Among (a) Original and Smoothed Curves, (b) Three Curves Having the Same Pattern but Different Levels and (c) Their DCT Coefficients, and (d) Reconstructed Curves by Inverse DCT with the First 14 Coefficients

representative values [9] have been used for the mass estimation of an impact object.

For finding a considerable feature vector representing the difference between APSD curves, this study proposes a process combining two methods: a smoothing technique and the discrete cosine transform. The extraction process is as follows. First, the APSD of an impact signal is calculated. Usually, the APSD contains too many small and narrow peak components, which cannot be easily utilized as a basic pattern in the frequency domain. Second, a rectangular smoothing technique [14] is applied to a raw APSD to simplify its curve shape. This is the simplest method to smoothen a curve. The smoothing was defined as follows:

$$
S_{i}=\frac{L_{i-2}+L_{i-1}+L_{i}+L_{i+1}+L_{i+2}}{5}
$$

where $\mathrm{L}_{i}$ is the $i^{\text {th }}$ level in the raw APSD, $\mathrm{S}_{i}$ is the $i^{\text {th }}$ level after smoothing, $i$ runs from 3 to $N-2$, and $N$ is the number of total data. Fig. 6(a) compares the smoothed APSD with the original APST. For better understanding, the example signal is sampled at a sampling frequency $\left(f_{s}\right), 102.4 \mathrm{kHz}$, and the following options are applied for the APSD calculation: 1024 data in the FFT, , 66\% overlap, and a rectangular window.

In Fig. 3, when the impact energy is changed for a fixed impact mass, the pattern of the APSD remains unchanged while its absolute level changes. In addition, the distance between the impact and measurement points could also affect the absolute level of the APSD. In order to obtain an optimal feature vector, it is necessary to extract only the pattern as a function of frequency regardless of the level difference. For this, the DCT is used in this study. The DCT is closely related to the discrete Fourier transform and is defined as follows [15]:

$$
\begin{gathered}
y_{k}=w(k) \sum_{i=1}^{N} S_{i} \cos \left(\frac{\pi(2 i-1)(k-1)}{2 N}\right) \\
w(k)= \begin{cases}\frac{1}{\sqrt{N}} & \text { for } k=1 \\
\sqrt{\frac{2}{N}} & \text { for } 2 \leq k \leq N\end{cases}
\end{gathered}
$$


where $\mathrm{y}_{k}$ is the $k^{\text {th }}$ DCT coefficient with $\mathrm{k}$ from 1 to $\mathrm{N}$. Fig. 6(b) shows that the three curves have identical patterns but their levels are different. Fig. 6(c) compares the DCT coefficients for the three curves in Fig. 6(b). All coefficients except the first are the same. The first coefficient is related to the absolute level, that is, the DC offset. Fig. 6(d) shows the reconstructed curves by the inverse DCT (iDCT) for the first 14 coefficients.

As shown in Fig. 6(d), the DCT has the advantage that the original curve can be reconstructed with only a few DCT coefficients. In order to determine how many coefficients are suitable to reconstruct the original curve, mean square errors (MSE) between the original and reconstructed curves were calculated as a function of the number of coefficients used for the reconstruction. It is shown in Fig. 7 that the original and reconstructed curves are quite similar when the number of coefficients used for the reconstruction is more than only 13. Judging from the above results, the DCT coefficients, excluding the first one, can be used as a good feature vector that represents the pattern of the APSD curve. Fig. 8 illustrates how to extract the DCT based feature vector.

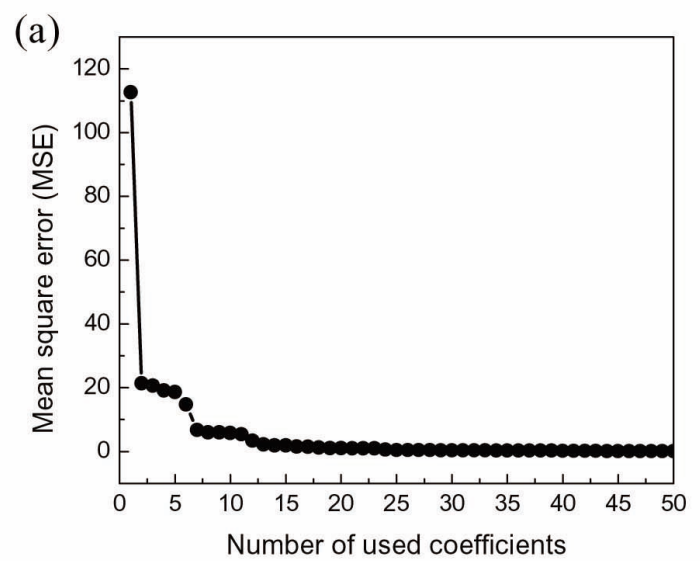

\subsection{Structure of the ANN Model}

In this study, an ANN model is designed with a feed forward network using error back-propagation training. The ANN model consists of 1 hidden and 1 output layer. Log-sigmoid and linear transfer functions are used for the neurons of the hidden and output layer, respectively. As shown in Fig. 9, this structure is the most basic and simplest for the ANN.

To deal with the mass estimation problem as a supervised problem, the output is the mass of an impact object, and the number of neurons in the output layer is then one. It is important to decide the number of hidden neurons in the design of the ANN model. If too many hidden neurons are taken, overestimation may occur; conversely, if the number of hidden neurons is not high enough, it is difficult to obtain expected performance. For this reason, in theory, the recommended number of hidden neurons $\left(n_{H}\right)$ is determined as follows [11]:

$$
n_{H}=\frac{\frac{n_{I} \times N_{I}}{10}-n_{O}}{n_{I}+n_{O}+1}
$$

Fig.7. (a) MSE between Original and Reconstructed Curves as a Function of the Number of Used Coefficients for the Reconstruction and (b) Comparison between Original and Reconstructed Curves
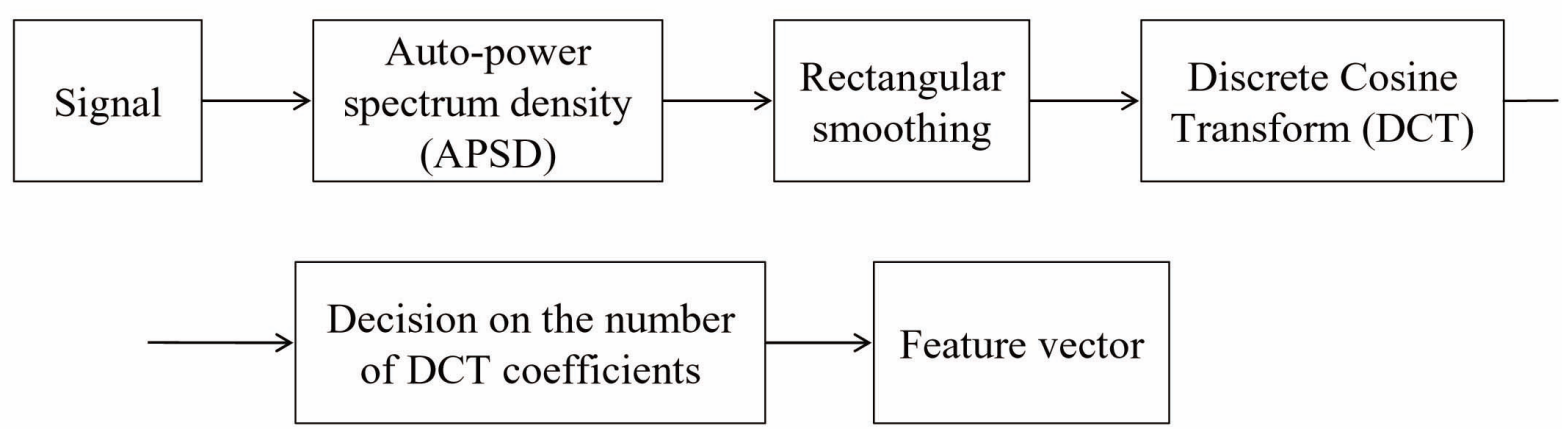

Fig.8. Flowchart Representing how to Extract the DCT Based Feature Vector for Input of the ANN Model 


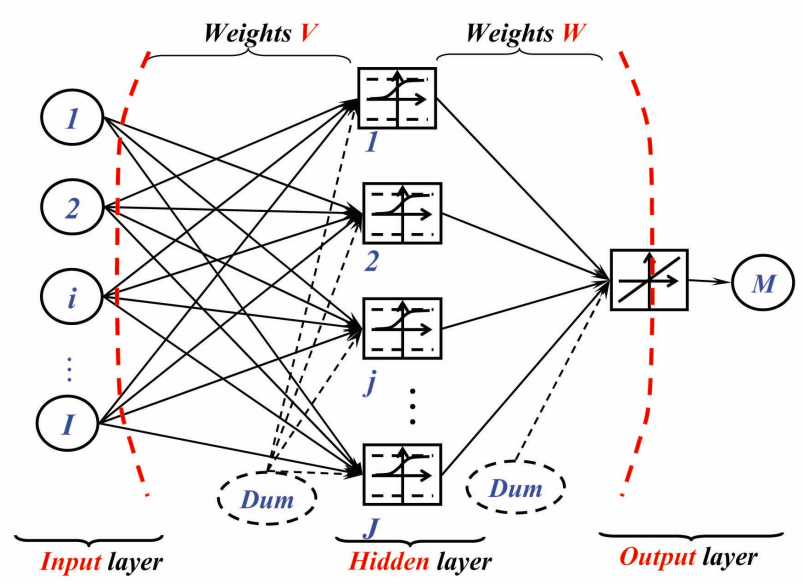

Fig. 9. ANN Structure Used for Estimating an Impact Mass in this Study

where $n_{I}$ is the number of input parameters, $N_{I}$ the number of total input data, and $n_{O}$ the number of output neurons. Unfortunately, this formula can only be used as a reference, because the number of hidden neurons is strongly dependent on the complexity of the problem. In this study, the final decision on the number of hidden neurons is made by trial and error based on the mean square error between real and estimated masses for training and test data for the ANN model and the recommended number.

\section{APPLICATIONS: MASS ESTIMATION}

\subsection{For a Large-Sized Steel Plate}

Mass estimation using the ANN model is applied to a large-sized steel plate $(2 \mathrm{~m} \times 2 \mathrm{~m} \times 0.01 \mathrm{~m})$. To obtain training data for the ANN model, 8 solid spheres with different masses, $112.7 \mathrm{~g}, 67.7 \mathrm{~g}, 36.9 \mathrm{~g}, 24.7 \mathrm{~g}, 17.7 \mathrm{~g}, 9.3 \mathrm{~g}$, $6.5 \mathrm{~g}$, and $3.1 \mathrm{~g}$, are collided against the plate. The impact energy is adjusted by releasing the ball at 5 different heights. The positions of the accelerometer and excitation are shown in Fig. 1(a). For the test data for the ANN model, 10 solid spheres are collided against the plate at arbitrary points with arbitrary energy. As a result, for each accelerometer, 200 impact signals for the training and 120 impact signals for the test are obtained and their DCT coefficients are calculated.

With the training data, the ANN model for each accelerometer is designed; that is, 8 ANN models are constructed. The effects of two nuisance variables, the transmission property of the structure and the position of sensor, on the shape of the APSD can e neglected, as the information is already included in the acceleration signals. Mass is estimated with each signal from every measure point by using the designed ANN models. The masses are then averaged to obtain a representative mass, which is the final output of the mass estimation using the ANN model.

In order to investigate the mass estimation performance according to the number of input parameters, the results obtained using 8, 13, and 20 DCT coefficients as inputs are compared. As already noted above, the first DCT coefficient related to the overall level is eliminated from the input parameter. From Fig. 7(b), it is seen that the reconstructed curve with 9 DCT coefficients is remarkably different from those with 14 and 21 DCT coefficients. Fig. 7(a) shows the quantitative differences; owing to these differences, three different numbers of DCT coefficients are chosen for comparison of estimation performance.

Fig. 10 shows the distribution of the estimated mass for training and test data used for the design of the ANN model. As shown in Figs. 10(a) and 10(b), the case using 8 DCT coefficients as input has relatively large deviations from the actual mass (the straight line). The deviations are especially dominant for the heavy mass, $112.7 \mathrm{~g}$. On the contrary, it is seen that the two other cases have quite similar estimation performances with each other in spite of the difference in the number of inputs of the ANN model.

In order to assess the extent of the deviation from the identical line in greater detail, relative errors between the actual and estimated masses in the test data is calculated. The relative error $\left(E_{\text {rel }}\right)$ was defined as follows:

$$
E_{r e l}=\frac{1}{N} \sum_{n=1}^{N} \frac{\left|m_{i}-M\right|}{M} \times 100(\%)
$$

Here, $m$ is the estimated mass, $M$ the actual mass, and $N$ the number of estimations. Fig. 11 compares the relative error as a function of the actual mass for different numbers of DCT coefficients used as input.

The case using 13 DCT coefficients has relative errors below $30 \%$ for all masses except the two lightest masses: $4.6 \mathrm{~g}$ and $6.5 \mathrm{~g}$. For these masses, all ANN models yielded large relative errors of more than $100 \%$. It is speculated that these errors are due to the light masses not sufficiently exciting the large-sized plate. In addition, the case using 20 DCT coefficients does not show substantial improvement in terms of the relative error compared to the case using 13 DCT coefficients. For some masses, the former case shows higher relative errors than the latter case. This means that 13 DCT coefficients are sufficient to represent the features of the APSD from the large sized plate and too many input parameters increase the complexity of the design of the ANN model. In the case of using 13 DCT coefficients as an input, the number of hidden neurons is about 14 depending on the ANN model.

To compare the performance of mass estimation by the ANN model, the FR method, one of the conventional methods for the mass estimation of loose parts, is also applied for an identical set of impact signals as used with the ANN model. For calculation of the FR value, low 
frequency components between $2 \mathrm{kHz}$ and $10 \mathrm{kHz}$ were chosen, since the first local peak appears in this frequency range, as shown in Fig.2, and high frequency components between $10 \mathrm{kHz}$ and $40 \mathrm{kHz}$ were chosen. As shown in
Fig. 12, the FR values widely overlapped for the different masses compared with the masses estimated by the ANN model. It is difficult to see the difference between FR values for the masses of $67.7 \mathrm{~g}$ and $112.7 \mathrm{~g}$. Judging from (a)

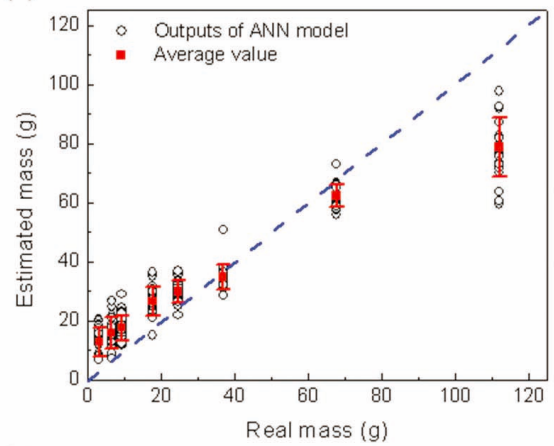

(b)

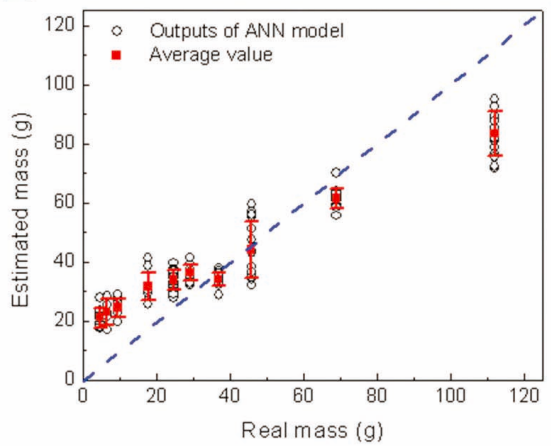

(c)

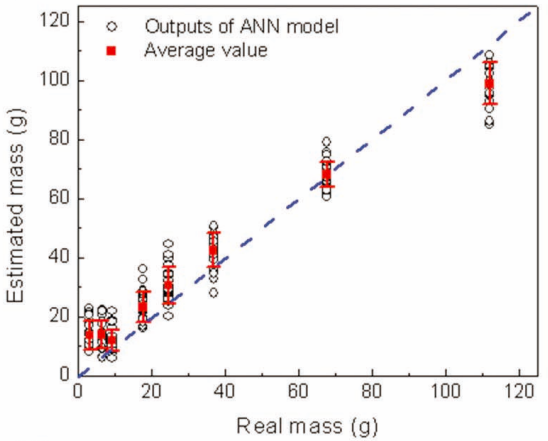

(d)

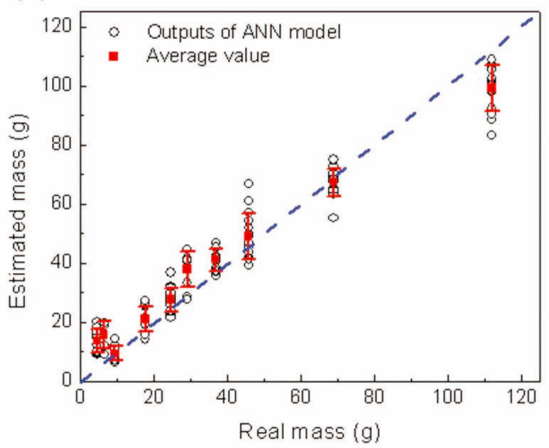

(e)

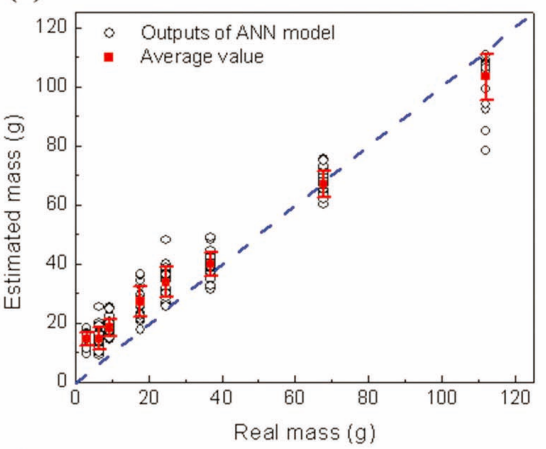

(f)

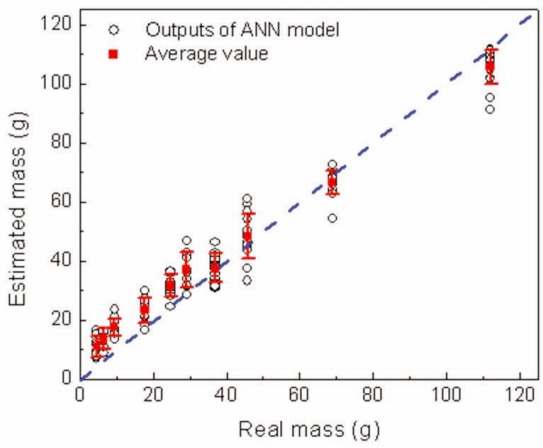

Fig.10.Comparisons between the Results of Mass Estimation by ANN Models with the Different Number of Input Parameters for the Plate: (a) Training and (b) Test Data with 8 DCT Coefficients, (c) Training and (d) Test Data with 13 DCT Coefficients, (e) Training and (f) Test Data with 20 DCT Coefficients. In the Figures, the Bar Indicates the Standard Deviation of Estimated Values for Each Mass and the Dashed Line is Points where Real and Estimated Masses are Identical with Each Other

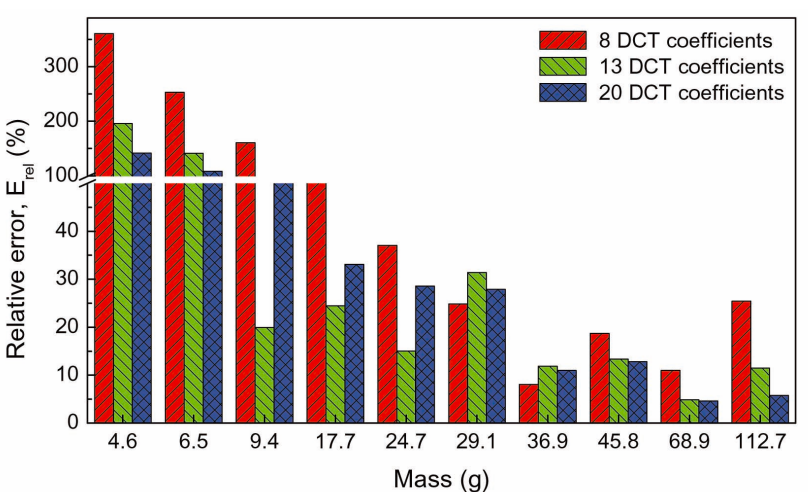

Fig.11. Comparison of the Relative Errors between Estimated and Real Masses for the Test Data Used for the Design of the ANN Model for the Plate as a Function of Real mass According to the Number of DCT Coefficients Used as Input Data

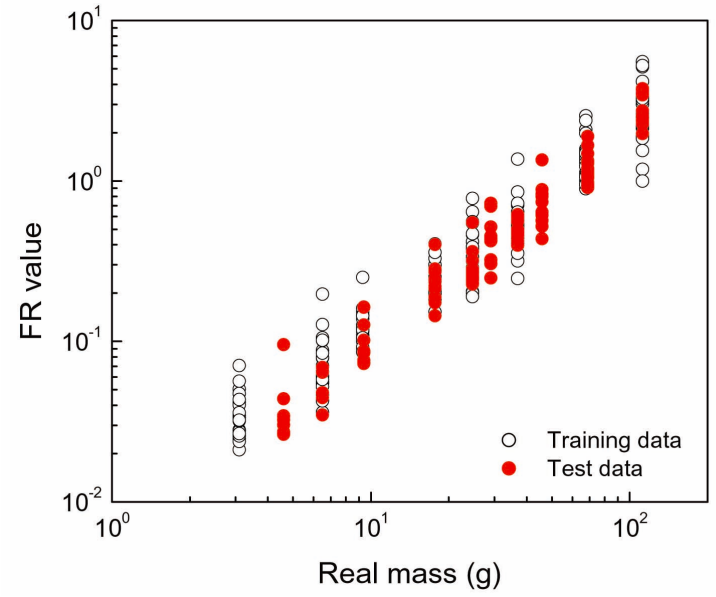

Fig. 12. FR Values of Impact Signals Used as Training and Test Data for the Design of ANN Model for the Plate 
this comparison, the ANN model provides better mass estimation performance than the FR method.

\subsection{For a 1/8-Scaled Mock-up}

Mass estimation using the ANN model is also applied to a $1 / 8$-scaled mock-up of a reactor pressure vessel (Fig. 13(a)). To obtain training data for the ANN model, 8 solid spheres with masses of $112.7 \mathrm{~g}, 68.9 \mathrm{~g}, 36.9 \mathrm{~g}, 29.1 \mathrm{~g}, 24.7 \mathrm{~g}$, $17.7 \mathrm{~g}, 9.4 \mathrm{~g}$, and $4.6 \mathrm{~g}$ are used. They are shot at 5 different points on the mock-up with 5 different energies for each sphere. 8 accelerometers are positioned on the surface of the bottom part of the mock-up, as shown in Fig. 13(b). The positions of 3 accelerometers are identical with the positions of sensors for loose part monitoring of the reactor pressure vessel after considering the reduced scale. The other accelerometers are arbitrarily located. To collect test data for the ANN model, 11 solid spheres are shot at arbitrary points with arbitrary energy. As a result, for each accelerometer, 200 impact signals for the training and 99 for the test were obtained and DCT coefficients were calculated.

First, Fig. 14 shows the effects of impact mass and energy on the APSD of the impact signal. As in the results for the plate, the variation of mass considerably affects the change in the pattern of the APSD curves. It appears that the energy difference also contributes somewhat to the pattern change. Second, the number of coefficients needed to reconstruct the pattern for the mock-up model is examined. Fig. 15(a) presents the results for the MSE between the original and reconstructed curves as a function of the number of coefficients used in the ANN model. It is shown that the use of more than 13 DCT coefficients (a)

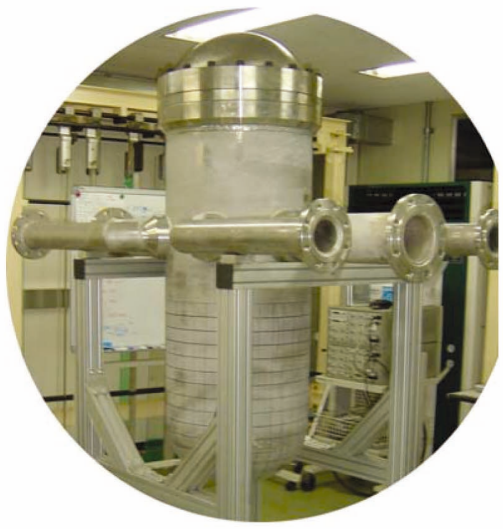

(b)

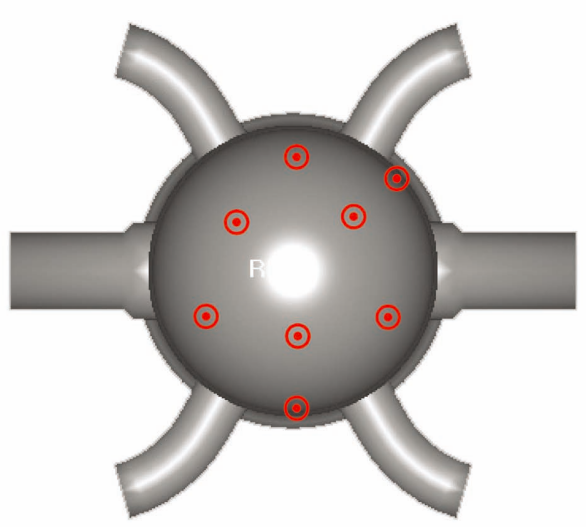

Fig.13. (a) 1/8-Scaled Mock-Up of a Reactor Pressure Vessel and (b) the Positions of 8 Accelerometers

(a)

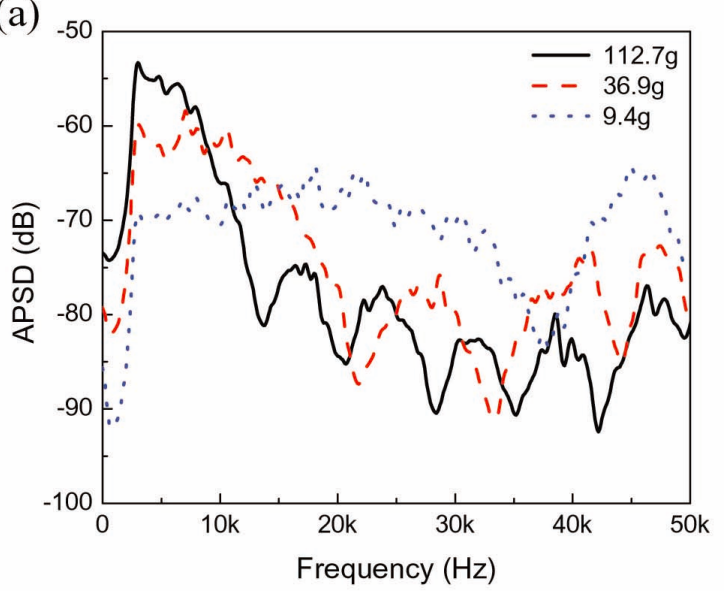

(b)

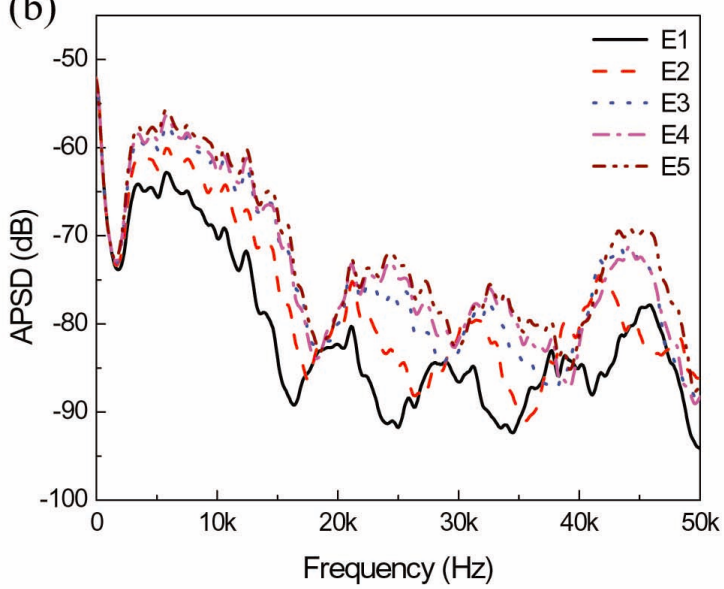

Fig.14. Comparisons of the Auto-power Spectrum Density (APSD) Curves According to (a) Impact Mass and (b) Impact Energy for the Mock-Up. In Fig. 14(b), Ascending Order of Energy: E1, E2, E3, E4, and E5 
including the first one can reconstruct the original APSD with high accuracy. Fig. 15(b) compares the original curve to reconstructed curves with different numbers of DCT coefficients.

For the mock-up, ANN models are constructed for each allocated accelerometer. The representative estimated mass is an average value of individual estimated masses obtained from the corresponding ANN models. In Fig. 15(a), 9 DCT coefficients are not enough to sufficiently represent the pattern of the APSD. Hence, the ANN models using 8 DCT coefficients excluding the first one as an input cannot correctly estimate the impact masses, as shown (a)

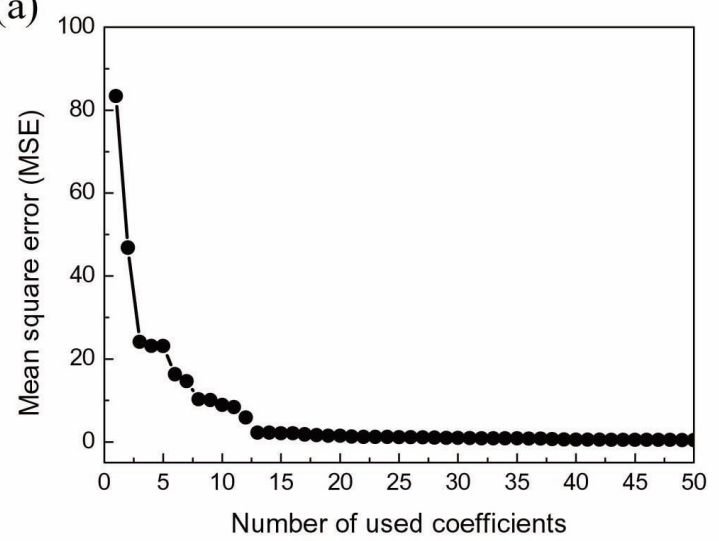

(b)

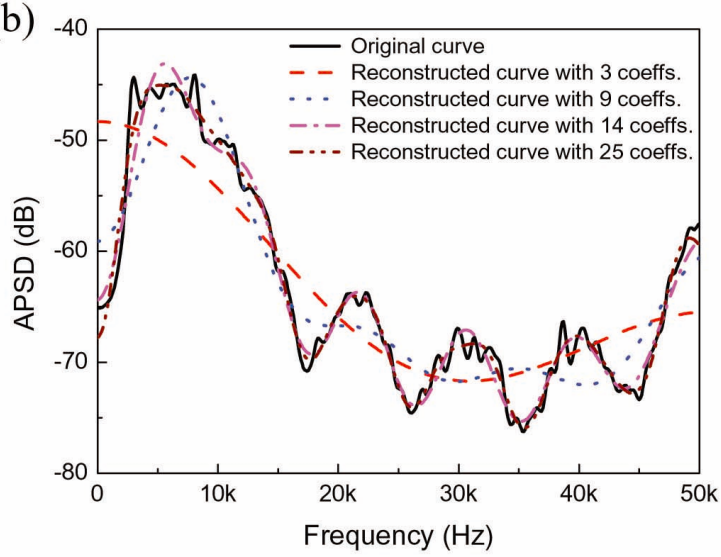

Fig.15. For the Mock-up, (a) MSE between Original and Reconstructed Curves as a Function of the Number of Coefficients Used for the Reconstruction and (b) Comparison between Original and Reconstructed Curves

(a)
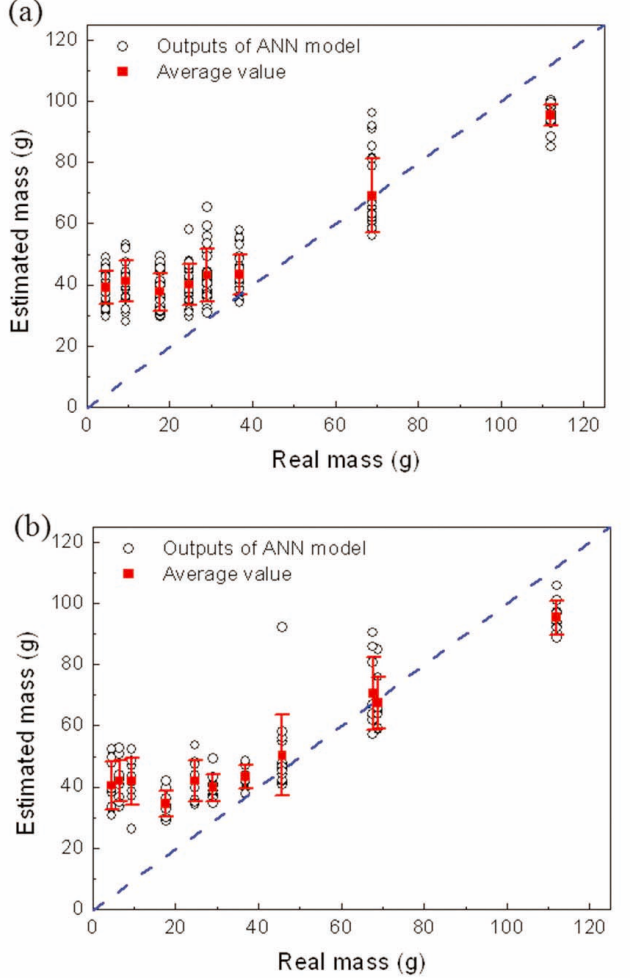

(c)

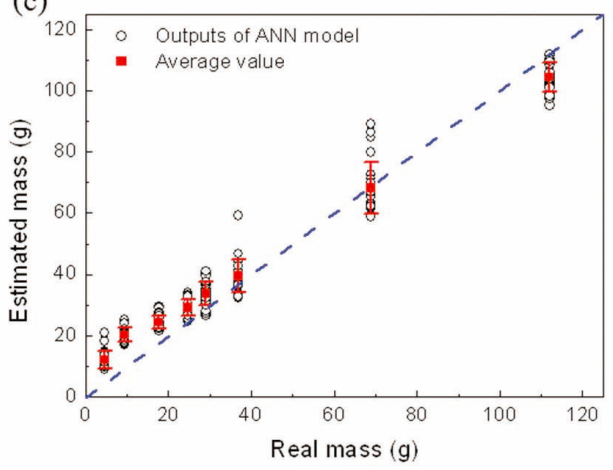

(d)

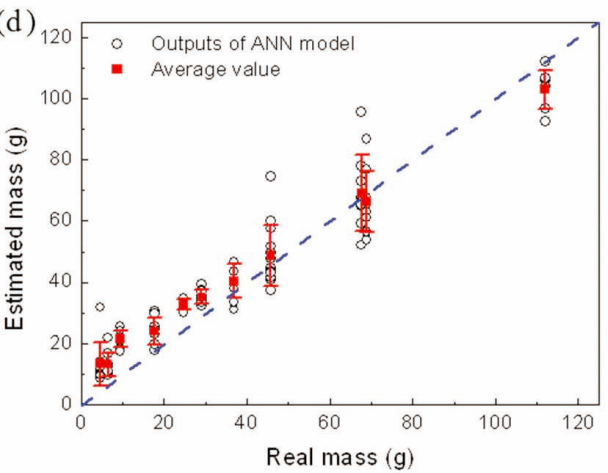

Fig. 16. Comparisons between the Results of Mass Estimation by ANN Models with Different Numbers of Input Parameters for the Mock-up: (a) Training and (b) Test Data with 8 DCT Coefficients, (c) Training and (d) Test Data with 13 DCT Coefficients. In the Figures, the Bar Indicates the Standard Deviation of Estimated Values for Each Mass and the Dashed Line is Points where the Real and Estimated Masses are Identical with Each Other 
in Figs. 16(a) and 16(b). In particular, for light masses, the deviation errors are large and it is difficult to distinguish between different masses due to the large overlap on the error bars. Moreover, the ranges of estimation for two relatively heavy masses, $68.9 \mathrm{~g}$ and $112.7 \mathrm{~g}$, partially overlapped each other. However, when 13 DCT coefficients excluding the first one are used for the ANN model, the estimation performance is remarkably improved. As shown in Figs. 16(c) and 16 (d), although the estimated values for a few masses overlapped, standard deviations for the estimated masses are much smaller than in the case of using only 8 DCT coefficients and the error ranges of the estimated masses are also much smaller. Fig. 17 shows that there is no appreciable improvement in the estimation performance when 25 DCT coefficients are used in comparison with the case of using 13 DCT coefficients. It can be concluded that 13 DCT coefficients are sufficient

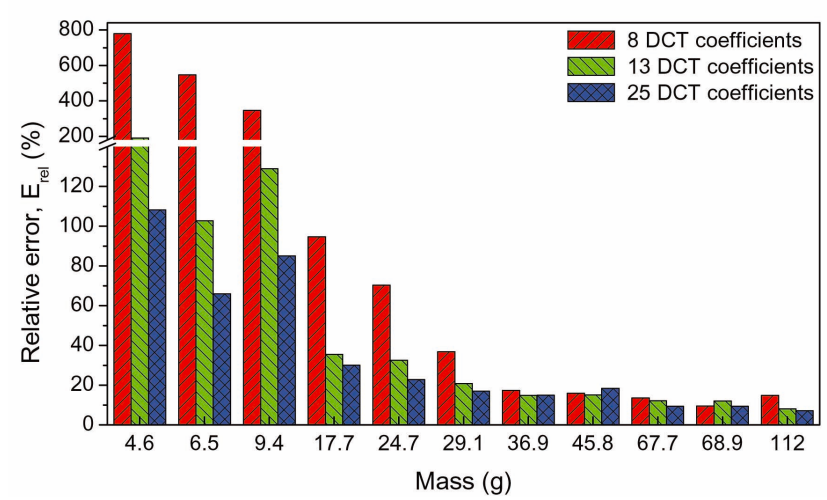

Fig.17. Comparison of Relative Errors between Estimated and Real Mass for the Test Data Used for the Design of the ANN Model for the Mock-up as a Function of Real Mass According to the Number of DCT Coefficients Used as Input Data

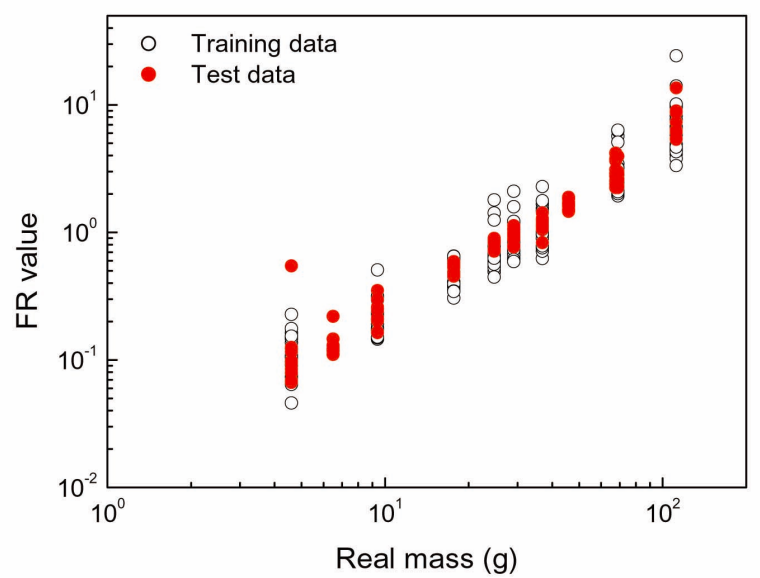

Fig. 18. FR Values of Impact Signals Used as Training and Test Data for the Design of the ANN Model for the Mock-up to represent the pattern of the APSD in this case. When 13 DCT coefficients are used as an input, the number of hidden neurons is about 16 , while it depends on the specific ANN model.

FR values on impact signals used for the design of the ANN model are also calculated in the same frequency ranges as in section 4.1 and the results are shown in Fig. 18. For each FR value, the range of corresponding mass is too wide and hence discrimination between two masses is practically impossible in comparison with mass estimation by the ANN model.

\section{CONCLUSION}

This study proposes a method using an ANN model to estimate the mass of a foreign object impacting a large-sized structure. Given the observation that the APSD of an impact signal is strongly affected by the mass of the impacting object, the pattern of the APSD is considered an important factor for the mass estimation. To quantitatively represent the pattern of the APSD curve, the DCT is introduced. Since the original curve can be reconstructed from only a few DCT coefficients, it is possible to precisely extract the features of the APSD curve and also decrease the number of input parameters for the ANN model. In addition, it is possible to represent the pattern of the APSD curves regardless of the difference in the absolute level by excluding the first DCT coefficient.

The proposed method using the ANN model is applied to two structures: a large-sized plate and a $1 / 8$-scaled mock-up of a reactor pressure vessel. As a whole, for all masses except for the light masses below $10 \mathrm{~g}$, relative errors between the real and estimated masses are below $30 \%$. Through comparison with the FR method it is shown that the proposed method improves the estimation performance. Moreover, the ANN model make it possible to directly obtain an estimated mass without any mapping between different domains, such as mapping the FR value to the mass domain. In addition, the method is free from the effects of the transmission properties of the structure and the sensor position.

While the proposed method is developed for mass estimation, when the difference between two masses is below a certain limit, the distribution ranges of estimated masses overlap extensively. A possible reason for this is that the APSD is only slightly changed even when the impact energy is varied. The current results were obtained under conditions without background noise. In order to improve the estimation performance of the proposed method and apply it to real field conditions in future works, it will be necessary to deal with several different in-field conditions: the characteristics of background noise, the effect of impact energy, coupling between the wall (solid) and internal fluid, and the shape of the loose part. 


\section{REFERENCES}

[1] J.-S. Kim, I.-K. Hwang, J.-T. Kim, and J. Lyou, "Development of automatic algorithm for localizing loose parts with a steam generator," Nuclear Engineering and Design, 219, 269 (2002).

[2] B. J. Olma, "Source Location and Mass Estimation in Loose Parts Monitoring of LWR's," Progress in Nuclear Energy, 15, 583 (1985).

[3] S. M. Ziola and M. R. Gorman, "Source location in thin plates using corss-correlation," J. Acoust. Soc. Am. 90, 2551 (1991).

[4] J.-H. Park and Y.-H. Kim, "Impact Source Localization on an elastic plate in a noisy environment," Meas. Sci. Technol., 17, 2757 (2006).

[ 5 ] D.-B. Yoon, J.-H. Park, Y.-C. Choi, H.-S. Kwon, and J.-H. Lee, "Enhancement of Impact Mass Estimation Algorithm for a Plate Type Structure," The Japanese Society for NonDestructive Inspection, 48, 1249 (2007).

[6] B. J. Olma, "Experience with Identification of Loose Parts by Acoustic Monitoring of Primary System," Progress in Nuclear Energy, 43, 225 (2003).

[7] C. W. Mayo, "Loose-part Mass and Energy Estimation,"
Progress in Nuclear Energy, 34, 263 (1999).

[8] T. Tsunoda, T. Kato, et al., "Studies on the Loose Part Evaluation Technique," Progress in Nuclear Energy, 15, 569 (1985).

[9] S. Figedy and G. Oksa, "Modern Methods of Signal Processing in the Loose Part Monitoring System," Progress in Nuclear Energy, 46, 253 (2005).

[10] T. Tsunoda, K. Sano, O. Tsuneoka, and T. Morioka, "Acceleration Signal Characteristics for Loose Part Impact," Journal of Nuclear Science and Technology, 23, 968 (1986).

[11] R. O. Duda, P. E. Hart, and D. G. Strok, Pattern Classification, New York, Join Wiley \& Sons, Inc. (2001).

[12] J. M. Zurada, Introduction to Artificial Neural Systems, New York, West Publishing Comp. (1992).

[13] J. Korbicz, J. M. Koscielny, Z. Kowalczuk, and W. Cholewa, Fault Diagnosis: Models, Artificial Intelligence, Application, Berlin, Springer-Verlag (2004).

[14] Tom O'Haver, An Introduction to Signal Processing in Chemical Analysis, http: // www.wam.umd.edu / toh / spectrum / TOC.html (2009) (Last access June 6, 2010).

[15] A. K. Jain, Fundamentals of Digital Image Processing, New Jersey, Prentice-Hall (1989). 